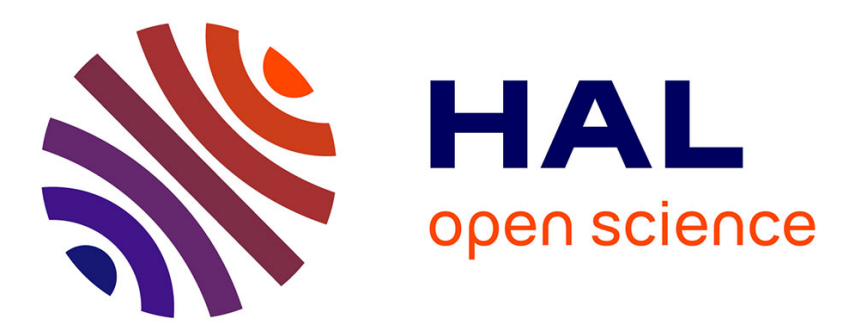

\title{
Préamplificateur de charge à faible bruit pour détecteurs au germanium compensé au lithium de grand volume sensible \\ P. Siffert, R. Regal
}

\section{- To cite this version:}

P. Siffert, R. Regal. Préamplificateur de charge à faible bruit pour détecteurs au germanium compensé au lithium de grand volume sensible. Revue de Physique Appliquée, 1968, 3 (2), pp.107-110. 10.1051/rphysap:0196800302010700 . jpa-00242831

\section{HAL Id: jpa-00242831 https://hal.science/jpa-00242831}

Submitted on 1 Jan 1968

HAL is a multi-disciplinary open access archive for the deposit and dissemination of scientific research documents, whether they are published or not. The documents may come from teaching and research institutions in France or abroad, or from public or private research centers.
L'archive ouverte pluridisciplinaire HAL, est destinée au dépôt et à la diffusion de documents scientifiques de niveau recherche, publiés ou non, émanant des établissements d'enseignement et de recherche français ou étrangers, des laboratoires publics ou privés. 


\title{
PRÉAMPLIFIGATEUR DE GHARGE A FAIBLE BRUIT POUR DÉTECTEURS AU GERMANIUM GOMPENSÉ AU LITHIUM DE GRAND VOLUME SENSIBLE
}

\author{
Par P. SIFFERT et R. REGAL, \\ Département de Physique des Rayonnements et d'Électronique Nucléaire, \\ Gentre de Recherches Nucléaires, Strasbourg-Cronenbourg.
}

(Reçu le 3 février 1968.)

\begin{abstract}
Résumé. - On décrit un préamplificateur de charge associé à un détecteur de rayons $\gamma$ au germanium compensé au lithium de grand volume sensible $\left(85 \mathrm{~cm}^{3}\right)$. Ce préamplificateur utilise comme élément d'entrée un transistor à effet de champ tétrode qui permet, lorsqu'il est refroidi à 150 oK, d'obtenir, avec le compteur mentionné, une résolution de $4,3 \mathrm{keV}$ pour un rayoncement $\gamma$ de $1,33 \mathrm{MeV}$.

Abstract. - A charge preamplifier, associated with a germanium-lithium $\gamma$-ray detector of large sensitive volume $\left(85 \mathrm{~cm}^{3}\right)$, is described. This preamplifier uses, as input element, a field effect tetrode transistor which allows, when cooled to $150 \mathrm{oK}$, to obtain, with the above counter, a $4.3 \mathrm{keV}$ FWHM for a $1.33 \mathrm{MeV} \gamma$-ray.
\end{abstract}

Les résolutions en énergie qu'il est possible d'atteindre avec les diodes au germanium compensé au lithium $[\mathrm{Ge}(\mathrm{Li})]$ sont très souvent limitées par le bruit du préamplificateur. Dans le cas de détecteurs de faible capacité, l'emploi de transistors à effet de champ (TEG) refroidis a permis d'obtenir des largeurs de raies à mi-hauteur nettement inférieures à $1 \mathrm{keV}$ pour des énergies de rayons $\gamma$ ou $\mathrm{X}$ de l'ordre de $100 \mathrm{keV}[1,2]$. Gette résolution se détériore rapidement (au moins de $50 \mathrm{eV} / \mathrm{pF}$ ) lorsque la capacité de la diode augmente. Cet effet peut être atténué en plaçant plusieurs transistors en parallèle dans l'étage d'entrée du préamplificateur (avec 4 TEG, par exemple, des variations de résolution de l'ordre de $15 \mathrm{eV} / \mathrm{pF}$ ont été trouvées). Néanmoins, cette solution présente deux inconvénients majeurs : la mise en parallèle de transistors augmente, comme on le verra plus loin, le bruit de l'ensemble et la détermination de la température optimale de fonctionnement de chaque TEC rend les réglages longs et délicats.

L'emploi de diodes $\mathrm{Ge}(\mathrm{Li})$ de structure coaxiale [3] de très grands volumes sensibles (de 80 à $100 \mathrm{~cm}^{3}$ ) nous a conduits à utiliser un préamplificateur adapté aux capacités élevées (souvent supérieures à $100 \mathrm{pF}$ ) de ces détecteurs et ne comportant qu'un transistor d'entrée.

I. Choix du transistor d'entrée. - Les performances des transistors à effet de champ, principalement à basse température, nous ont amenés à les employer comme élément d'entrée du préamplificateur.
Les sources principales de bruit à l'entrée sont constituées par le transistor à effet de champ et les résistances. D'après l'analyse de Van der Ziel, le bruit thermique dans le canal conducteur du TEG dont la largeur est modulée est prédominant. La valeur quadratique moyenne de bruit (exprimée en charges équivalentes à l'entrée) à la sortie d'un amplificateur comportant $n$ TEC en parallèle et des constantes de temps $\tau$ d'intégration et de différentiation identiques, est donnée approximativement par la relation :

$$
\overline{Q^{2}}=\frac{K T(\Sigma C)^{2}}{n g_{\mathrm{m}} \tau} \quad \text { avec } \quad(\Sigma C)^{2}=\left(C_{\mathrm{D}}+n C^{\prime}\right)^{2}
$$

dans laquelle $K$ désigne une constante, $T$ la température absolue, $g_{\mathrm{m}}$ la pente des transistors, $\Sigma C$ la capacité totale d'entrée, $C_{\mathrm{D}}$ et $C^{\prime}$ celle du détecteur et celle d'entrée d'un TEG.

Pour des détecteurs de grand volume $\left(C_{\mathrm{D}} \gg n C^{\prime}\right)$, l'augmentation du bruit en fonction de la capacité est donc :

$$
\frac{\mathrm{d} Q}{\mathrm{~d} C}=K^{\prime}\left(\frac{T}{n g_{\mathrm{m}}}\right)^{\mathbf{1} / \mathbf{2}} \quad\left(K^{\prime} \text { constante }\right) .
$$

Les paramètres essentiels dans le cas qui nous intéresse sont donc la pente $g_{\mathrm{m}}$ et la température de fonctionnement (le refroidissement améliore également la pente). Les TEC généralement utilisés dans les préamplificateurs (de type $2 \mathrm{~N} 3819,2 \mathrm{~N} 3823,2 \mathrm{~N} 4416$ ) ont des pentes voisines de $5 \mathrm{~mA} / \mathrm{V}$ pour des capacités d'entrée inférieures à $10 \mathrm{pF}$. Le TEG tétrode TIX S 35 


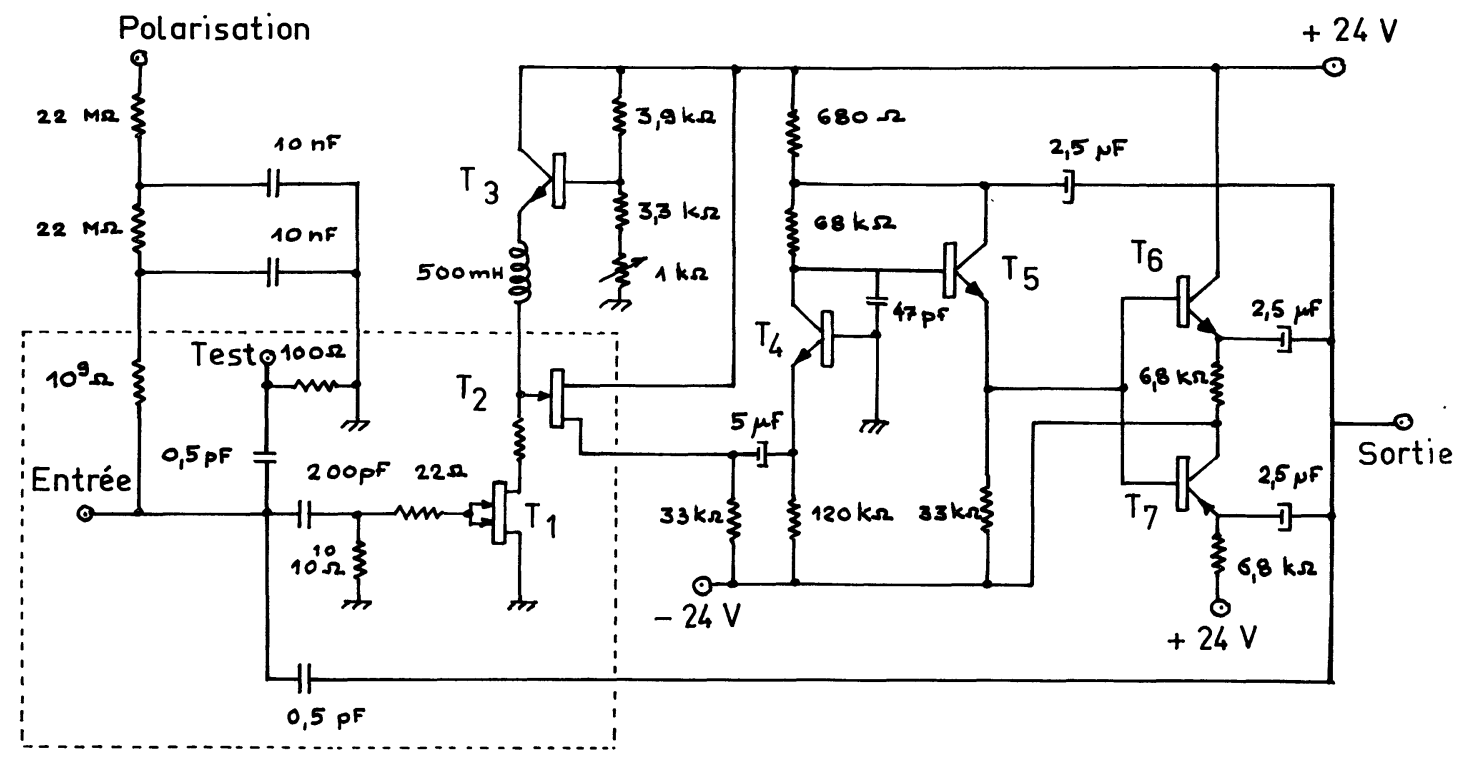

FIG. 1. $-\mathrm{T}_{1}=$ TIX S 35; $\mathrm{T}_{2}=2 \mathrm{~N} \mathrm{3823;} \mathrm{T}_{3}=2 \mathrm{~N} \mathrm{1613;} \mathrm{T}_{4}, \mathrm{~T}_{5}, \mathrm{~T}_{6}=2 \mathrm{~N} 3904 ; \quad \mathrm{T}_{7}=2 \mathrm{~N} 3906$.

par contre présente une valeur de $g_{\mathrm{m}}$ voisine de $20 \mathrm{~mA} / \mathrm{V}$ pour une capacité d'entrée de $12 \mathrm{pF}$. Le facteur de mérite très grand de ce TEG nous a conduits à le retenir comme élément d'entrée du préamplificateur.

II. Préamplificateur. - Le schéma du préamplificateur est représenté sur la figure 1. Comme indiqué plus haut, l'élément d'entrée est formé d'un transistor tétrode TIX S 35. Le deuxième étage est constitué par un transistor $2 \mathrm{~N} 3823$ monté en source asservie. La très faible impédance de sortie permet de monter éventuellement ces deux transistors et les résistances de charge directement dans le cryostat en vue de leur refroidissement. Les étages suivants sont empruntés à Smith et Cline [4]. Une contre-réaction capacitive $(0,5 \mathrm{pF})$ rend ce préamplificateur sensible à la charge.

Deux versions de ce préamplificateur, avec ou sans refroidissement de l'étage d'entrée, ont été réalisées.

III. Performances. - Fonctionnement a tempéRATURE AMBIANTE. - La contribution de l'amplificateur à la résolution de l'ensemble a été déterminée pour différentes capacités placées à l'entrée, en mesurant avec un voltmètre efficace le niveau de bruit à la sortie; la largeur de raie (en $\mathrm{keV}$ ) équivalente à cette tension de bruit a été obtenue à l'aide d'un compteur $\mathrm{Ge}(\mathrm{Li})$ et d'une source de rayonnement $\gamma$ d'énergie connue. Les résultats résumés dans le tableau ci-dessous sont représentés sur la figure 2 sur laquelle on a également reporté les valeurs trouvées en utilisant

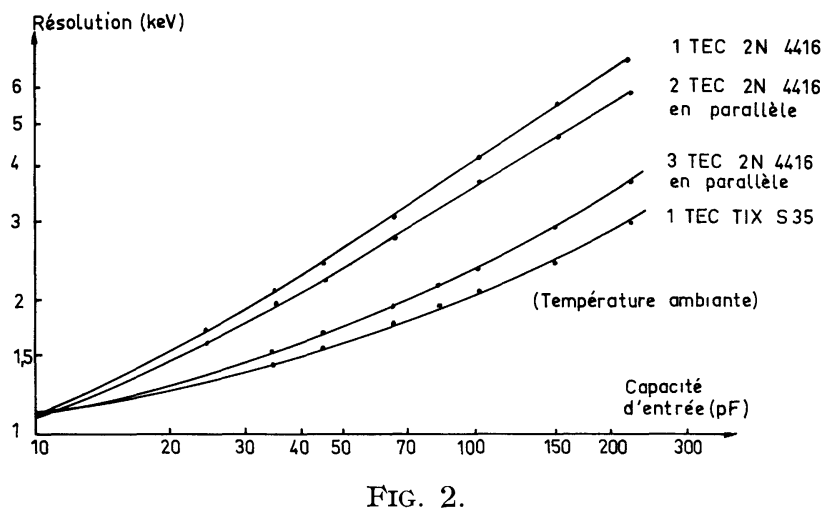

TABLEAU I

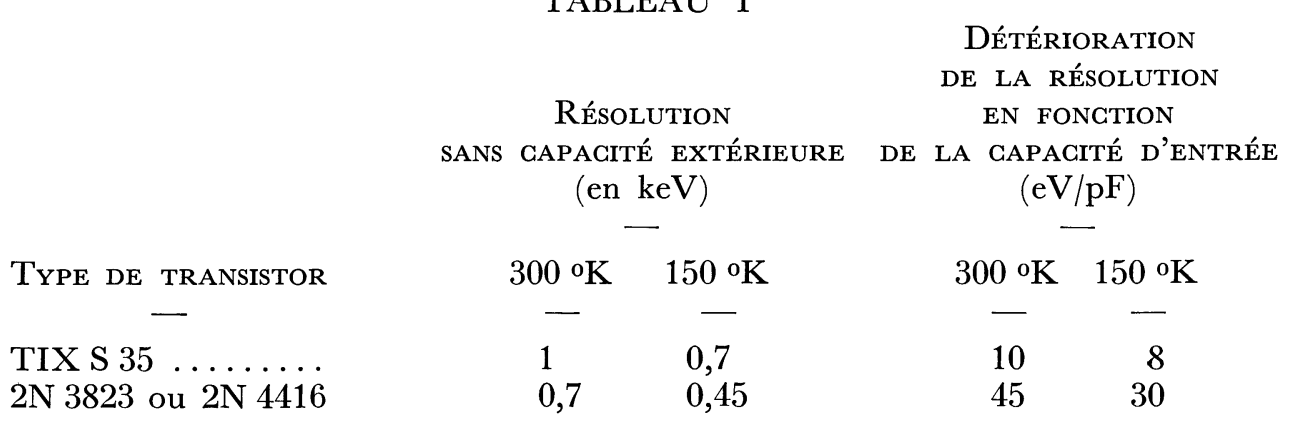


dans l'étage d'entrée un ou plusieurs (en parallèle) TEG sélectionnés de type $2 \mathrm{~N} 4416$.

Les performances du préamplificateur ont été ensuite examinées avec trois compteurs $\mathrm{Ge}(\mathrm{Li})$ de volumes voisins de 20,22 et $85 \mathrm{~cm}^{3}$ et de capacités respectivement égales à 56,60 et $125 \mathrm{pF}$, pour des tensions de l'ordre de 1000 volts. Dans ce but, on a déterminé la valeur commune optimale des constantes d'intégration et de différentiation pour les divers détecteurs. Les résultats obtenus avec la diode de $22 \mathrm{~cm}^{3}$ et le rayonnément $\gamma$ de ${ }^{137} \mathrm{Cs}$ ont été reportés sur la figure 3 en même temps que les largeurs à mi-hauteur observées, dans les mêmes conditions, en

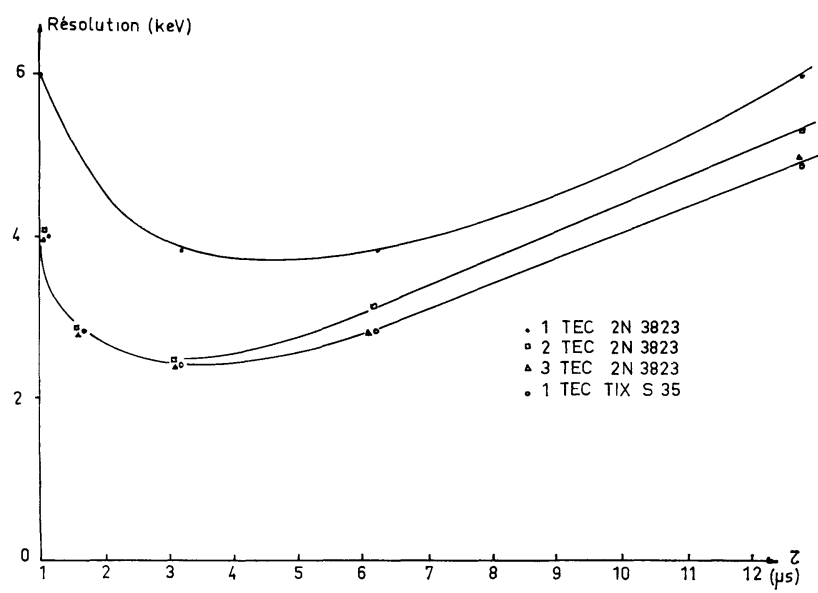

FIG. 3.

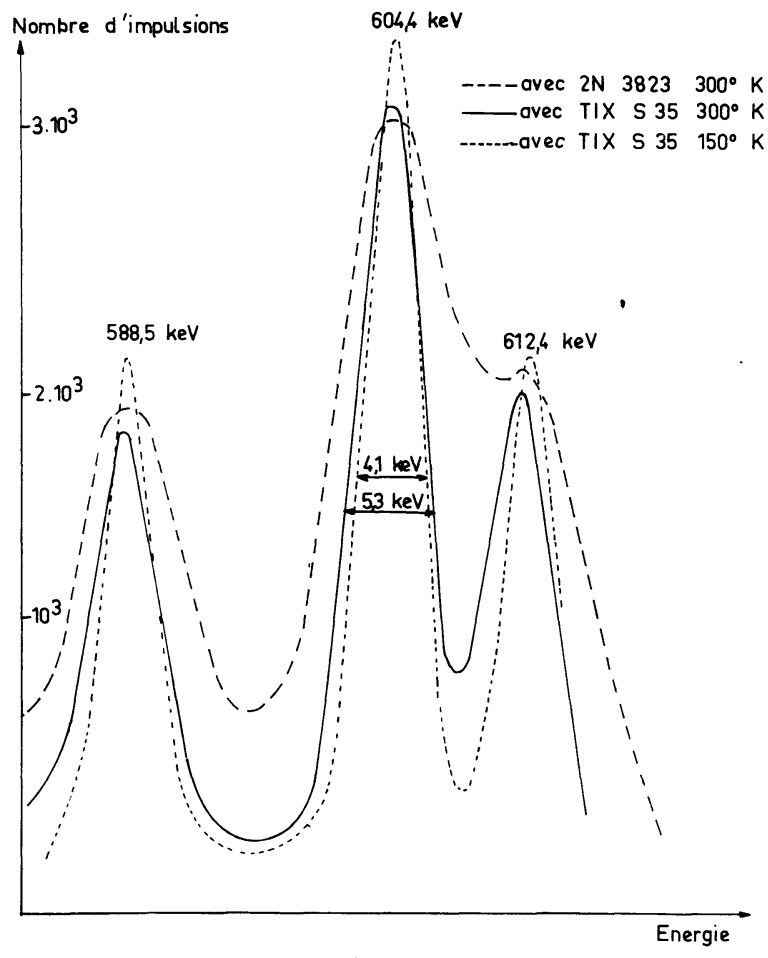

FIG. 4. remplaçant le transistor TIX S 35 par un ou plusieurs TEG $2 \mathrm{~N} 3823$. On remarquera que, pour les mêmes valeurs des constantes de temps, il est nécessaire de placer dans l'étage d'entrée 3 TEC en parallèle pour obtenir une résolution identique à celle que donne un montage à un seul transistor tétrode.

L'avantage de l'emploi du TEC tétrode apparaît nettement sur la figure 4 représentant une partie du spectre $\gamma$ de ${ }^{192}$ Ir enregistré à l'aide du compteur de $85 \mathrm{~cm}^{3}$ de volume sensible, polarisé sous 1000 volts et associé à un préamplificateur utilisant comme transistor d'entrée soit un TEC $2 \mathrm{~N} 4416$, soit un TEG TIX S 35.

Fongtionnement a BASSE température $\left(150^{\circ} \mathrm{K}\right)$. - Lorsque les deux transistors d'entrée (fig. 1) sont refroidis à $150{ }^{\circ} \mathrm{K}$, les performances du préamplifica-
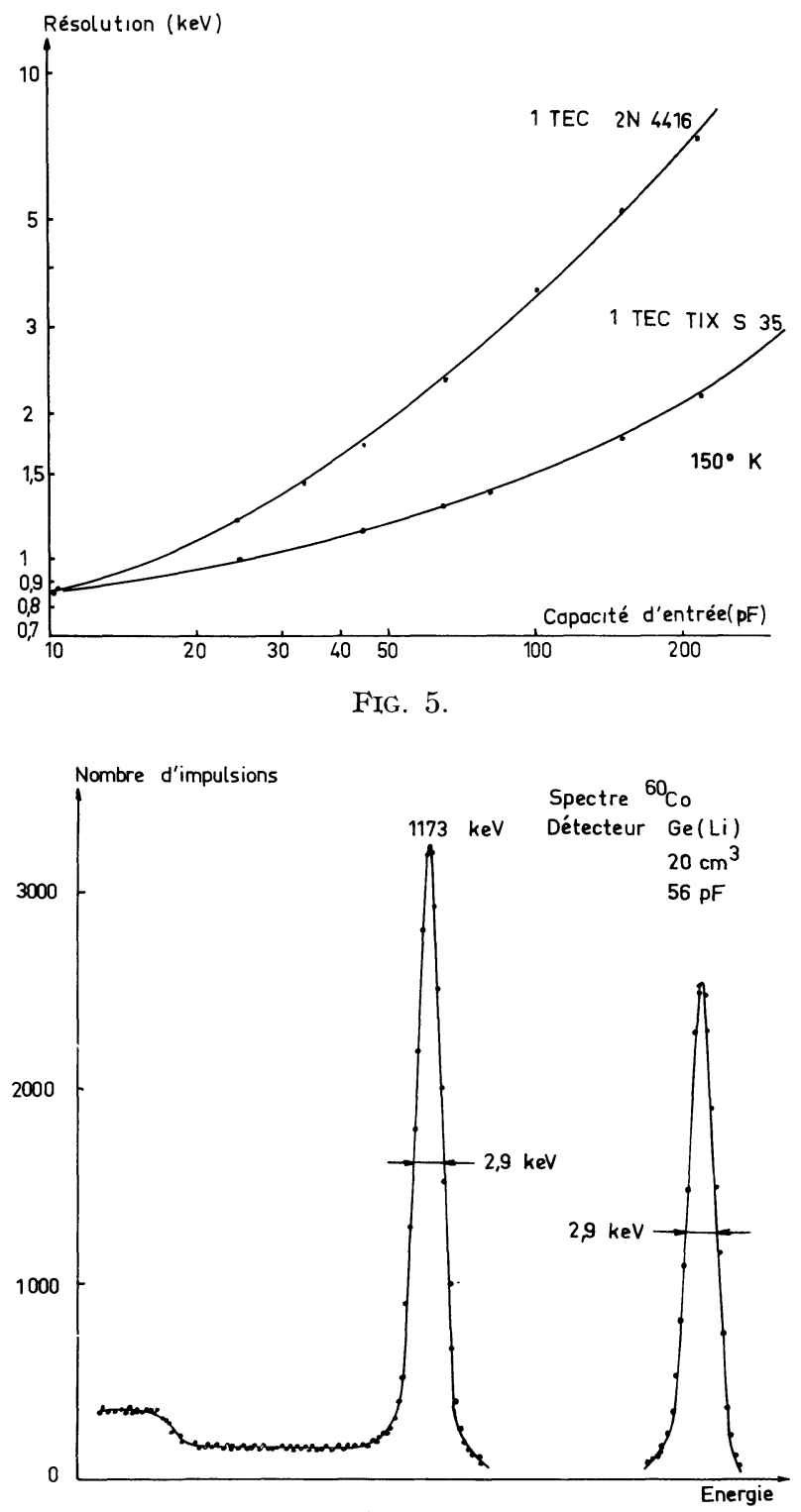

FIG. 6. 


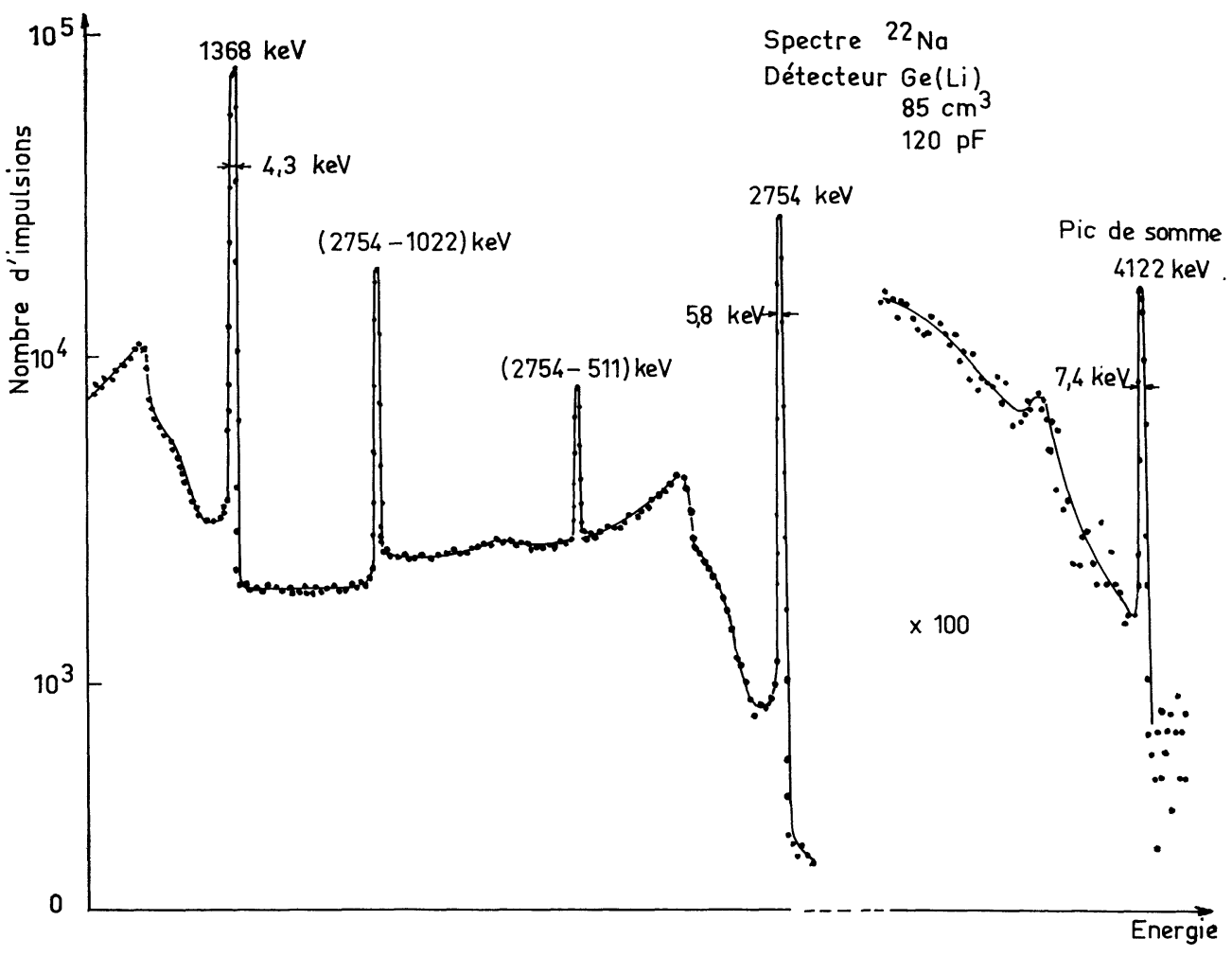

FIG. 7.

teur sont sensiblement améliorées. Nous avons repris dans ces conditions, avec les mêmes détecteurs, les essais décrits ci-dessus. Les résolutions trouvées sont indiquées dans le tableau I et la figure 5 montre la variation de la résolution en fonction de la capacité placée à l'entrée.

Sur les figures 6 et 7, nous avons représenté deux spectres enregistrés avec les diodes de 85 et de $22 \mathrm{~cm}^{3}$. On notera que l'efficacité importante du compteur de $85 \mathrm{~cm}^{3}$ permet l'observation de la raie somme dans le spectre de ${ }^{24} \mathrm{Na}$.

Plusieurs préamplificateurs de ce type dont les impulsions de sortie ont des temps de montée égaux à 13 ou $35 \mathrm{~ns}$, suivant que la capacité d'entrée est nulle ou égale à $100 \mathrm{pF}$, sont en fonctionnement au laboratoire depuis plus d'un an, notamment dans les expériences de spectrométrie $\gamma$. Leur stabilité les a également fait retenir pour des expériences de longuedurée.

\section{BIBLIOGRAPHIE}

[1] Heath (R. L.), BLACK (W. W.) et Cline (J. E.), I.E.E.E. Trans. Nucl. Sci., 1966, NS 13, no 3, 445.

[2] HARRIS (R. J.) et SCHULER (W. B.), Nucl. Instr. and Meth., 1967, 51, 341.
[3] Henck (R.), SiffeRT (P.) et CoChe (A.), Nucl. Instr. and Meth. (sous presse).

[4] SMITH (K. F.) et CLINE (J. E.), I.E.E.E. Trans. Nucl. Sci., 1966, NS 13, no 3, 468. 